\title{
Muscle activation varies between high-bar and low-bar back squat
}

\author{
Michal Murawa ${ }^{\text {Corresp., } 1}$, Anna Fryzowicz ${ }^{1}$, Jaroslaw Kabacinski ${ }^{1}$, Jakub Jurga ${ }^{1}$, Joanna Gorwa ${ }^{1}$, Manuela Galli ${ }^{2}$, \\ Matteo Zago ${ }^{2}$ \\ 1 Department of Biomechanics, Poznan University of Physical Education, Poznan, Poland \\ 2 Dipartimento di Elettronica, Informazione e Bioignegneria, Politecnico di Milano, Milano, Italy \\ Corresponding Author: Michal Murawa \\ Email address: murawa@awf.poznan.pl
}

Background Differences in the muscular activity between the high-bar back squat (HBBS) and the lowbar back squat (LBBS) on the same representative group of experienced powerlifters are still scarcely investigated. The main purpose of the study was to compare the normalized bioelectrical activity and maximal angles within single homogeneous group between the HBBS and LBBS for $60 \%$ one repetition maximum (1RM), 65\% 1RM and 70\% 1RM. Methods Twelve healthy men (age $24.3 \pm 2.8$ years, height $178.8 \pm 5.6 \mathrm{~cm}$, body mass $88.3 \pm 11.5 \mathrm{~kg}$ ), experienced in powerlifting performed HBBS and LBBS with comparable external loads equal 60\% 1RM, 65\% 1RM, and 70\% 1RM. Electromyography (EMG) signals of muscle groups were synchronously recorded alongside kinematic data (joints angle) by means of a motion capture system. Results EMG activity during eccentric phase of squat motion were significantly higher during LBBS than in HBBS for all selected muscles $(60 \% 1 \mathrm{RM}$ and $65 \% 1 \mathrm{RM})(p<0.05)$. All examined muscles were more activated during concentric phase of the squat cycle $(p<0.05)$. In the concentric phase, significant differences between the loads were generally not observed between just $5 \%$ 1RM change in load level for LBBS. Conclusions Our results confirmed significant differences in muscles activation between both squat techniques. Muscle activity during eccentric phase of squat motion were significantly higher during LBBS than HBBS. The differences are crucial for posterior muscle chain during eccentric phase of squat cycle. 
1 Muscle activation varies between high-bar and low-bar 2 back squat

3

4 5 6

7 10

Michal Murawa ${ }^{1}$, Anna Fryzowicz ${ }^{1}$, Jaroslaw Kabacinski ${ }^{1}$, Jakub Jurga ${ }^{1}$, Joanna Gorwa ${ }^{1}$, Manuela Galli², Matteo Zago ${ }^{2}$

${ }^{1}$ Department of Biomechanics, Poznan University of Physical Education, Poznan, Poland

${ }^{2}$ Dipartimento di Elettronica, Informazione e Bioignegneria, Politecnico di Milano, Milano, Italy

Corresponding Author:

Michal Murawa ${ }^{1}$

Krolowej Jadwigi 27/39, Poznan, 61-871, Poland

Email address: murawa@awf.poznan.pl

\section{Abstract}

\section{Background}

Differences in the muscular activity between the high-bar back squat (HBBS) and the low-bar back squat (LBBS) on the same representative group of experienced powerlifters are still scarcely investigated. The main purpose of the study was to compare the normalized bioelectrical activity and maximal angles within single homogeneous group between the HBBS and LBBS for $60 \%$ one repetition maximum (1RM), $65 \% 1 \mathrm{RM}$ and $70 \% 1 \mathrm{RM}$.

\section{Methods}

Twelve healthy men (age: $24.3 \pm 2.8$ years, height $178.8 \pm 5.6 \mathrm{~cm}$, body mass $88.3 \pm 11.5 \mathrm{~kg}$ ), experienced in powerlifting performed HBBS and LBBS with comparable external loads equal $60 \% 1 \mathrm{RM}, 65 \% 1 \mathrm{RM}$, and 70\% 1RM. Electromyography (EMG) signals of muscle groups were synchronously recorded alongside kinematic data (joints angle) by means of a motion capture system.

\section{Results} EMG activity during eccentric phase of squat motion were significantly higher during LBBS than in HBBS for all selected muscles $(60 \% 1 \mathrm{RM}$ and $65 \% 1 \mathrm{RM})(p<0.05)$. All examined muscles were more activated during concentric phase of the squat cycle $(p<0.05)$. In the concentric phase, significant differences between the loads were generally not observed between just 5\% 1RM change in load level for LBBS.

\section{Conclusions}

Our results confirmed significant differences in muscles activation between both squat techniques. Muscle activity during eccentric phase of squat motion were significantly higher 
39

40

41

42

43

44

45

46

47

48

49

50

51

52

53

54

55

56

57

58

59

60

61

62

63

64

65

66

67

68

69

70

71

72

73

74

75

76

77

78

during LBBS than HBBS. The differences are crucial for posterior muscle chain during eccentric phase of squat cycle.

\section{Introduction}

The squat is considered one of the most common strength and conditioning exercises in numerous sport disciplines for both professional and amateur athletes. The main reason for that is the existence of strong association between the squat maximum repetition and increased performance in various athletic tasks. The squat is used primarily to improve muscle strength and power performance of the hip and knee extensors and it is even more effective when performed with external load such as barbell (Wisloff et al., 2004; Usui et al., 2016; Wirth et al., 2016). Due to its multi-joint characteristics, it is also recognized as a screening test for movement deficits (Kritz, Cronin \& Hume, 2009; Myer et al., 2014; Kushner et al., 2015; Rabin \& Kozol, 2017) or even physical examination (Ahankoob et al., 2011). Different aspects of squat technique, including muscle activity, were subjects of many research projects and biomechanical analysis (Gullett et al. 2009; Schwanbeck, Chilibeck \& Binsted, 2009; McBride et al., 2010; Fujita et al., 2011; Bryanton et al., 2012; Clark, Lambert \& Hunter, 2012; Contreras et al., 2015; Contreras et al., 2016; Saeterbakken, Andersen \& van den Tillaar, 2016; Hammond et al., 2019). Among the groups which are most interested in the practical application of such results are powerlifters and their coaches. This is due to their competition goal which is lifting one repetition maximum (1RM) weight, so any tip such as training or lifting technique modifications that can improve performance can become crucial.

Powerlifters perform two main techniques of back squat with weights during their trainings: the high-bar back squat (HBBS) and the low-bar back squat (LBBS) (Wretenberg, Feng \& Arborelius, 1996; Glassbrook et al., 2019). The names of the variations describe the barbell position which is held either at the top of the trapezius muscle (just below the process of the $\mathrm{C} 7$ vertebra) (HBBS) or further down on the back along the spine of the scapula and over the posterior deltoid (LBBS) (Wretenberg, Feng \& Arborelius, 1996; Glassbrook et al., 2017). Even if LBBS usually allows to lift heavier loads, the HBBS still remains one of the most important exercise in athletes training. The LBBS is characterized by more forward torso position, decreased moment arm due to placing the bar lower on the back and higher activation of posterior muscles group (Glassbrook et al., 2017). The benefits of HBBS are more upright torso position, greater ranges of motion for ankle and knee joints which could result in higher activation of quadriceps muscles (Glassbrook et al., 2017). However, it is still not well evidenced what are the differences in muscular activity of lower part of the body between HBBS and LBBS (Glassbrook et al., 2017). To date, researchers did not analyze the differences in muscle activity between the HBBS and LBBS on the same representative group of experienced powerlifters.

The main aim of this study was to verify existing differences in electromyography (EMG) signal data for selected muscles involved in HBBS and LBBS performance and provide some strong evidence on muscular activation differences between the two squat variations. Authors expect to 
84

85

86

87

88

89

90

91

92

93

94

95

96

97

98

99

100

101

102

103

104

105

106

107

108

109

110

111

112

113

114

115

116

117

118

confirm that LBBS technique is more efficient than HBBS for posterior muscles of lower extremities, but also want to assess the scale of existing differences in experienced homogeneous group of powerlifters. The additional aim was to evaluate the influence of barbell weight level on selected muscle activity and verify if $5 \%$ change of load is enough to notice significant muscle activity differences in LBBS or HBBS.

\section{Methods}

\section{Participants}

Twelve healthy men (age: $24.3 \pm 2.8$ years, height $178.8 \pm 5.6 \mathrm{~cm}$, body mass (BM) $88.3 \pm 11.5$ $\mathrm{kg}$, BMI $27.5 \pm 2.7 \mathrm{~kg} / \mathrm{m}^{2}$ ) were selected to participate in the experiment. Seven of them were competitive powerlifters (National Academic Championships) and five of them were preparing for their first competition. They were classified as experienced in resistance training and performing squats (training experience $5.0 \pm 1.7$ years, $1 \mathrm{RM}_{\mathrm{HBBS}} / \mathrm{BM} 1.6 \pm 0.3,1 \mathrm{RM}_{\mathrm{LBBS}} / \mathrm{BM}$ $1.7 \pm 0.2$ ) (Earp et al., 2016; Banyard et al., 2017; Shariat et al., 2017; Hammer, Linton \& Hammer, 2018). 1RM for HBBS and LBBS were set separately during training sessions (one week apart). All participants did not experience any injury incidents in the previous two years, they refrained from lower body training for 48 hours before testing and they were able to squat with maximal effort. This project was approved by the Bioethical Committee of the Poznan University of Medical Sciences (number 546/16) and all subjects gave written informed consent to participate in this study.

\section{Experimental procedures}

The experiment was performed in three sessions (one week apart). The first was planned to test participants HBBS 1RM, the second to test LBBS 1RM and the third to evaluate powerlifters muscle activation during HBBS and LBBS with comparable relative external loads equal to $60 \%$, $65 \%$ and $70 \%$ of subjects' 1 RM. External load level was limited to $70 \%$ of $1 \mathrm{RM}$ in order not to interfere with individual training preparations to the National Academic Championships (Issurin, 2010) and also to allow athletes perform their optimum technique. 1 RM testing was followed after 5 minutes of general warm-up and stretching exercises. The athletes performed 8 repetitions at approximately $50 \%$ of $1 \mathrm{RM}$ followed by 3 repetitions at $70 \%$ of $1 \mathrm{RM}$ and then single repetitions with gradually heavier loads until failure. 1RM testing was consistent with acknowledged guidelines as described by Niewiadomski et al. (2008). All participants performed their squats in standardized powerlifting shoes (adidas powerlift 3).

The third session (lasting approximately two hours) started from preparations for EMG data collecting in accordance with SENIAM recommendations (Kasman et al., 1998; Hermens et al., 2000). Before electrodes placement, the skin area was cleaned with alcohol and shaved if needed. Pairs of $\mathrm{Ag} / \mathrm{AgCl}$ electrodes (SORIMEX, Poland, $1 \mathrm{~cm}$ diameter), were placed bilaterally in a bipolar configuration along the longitudinal axis of lumbar erector spinae (LES), gluteus maximus (GM), long head of biceps femoris (BF), rectus femoris (RF), vastus lateralis (VLO) and vastus medialis (VMO). 
119 The inter-electrode distance (center to center) was $2 \mathrm{~cm}$. Proper placement was confirmed with

120

121

122

123

124

125

126

127

128

129

130

131

132

133

134

135

136

137

138

139

140

141

142

143

144

145

146

147

148

149

150

151

152

153

154

155

156

157

158

manual muscle testing and visual inspection of the raw EMG signal. The ground electrode was placed over the posterior superior iliac spine. A set of 19 reflective markers were then fixed by the same investigator on anatomical landmarks according to Vaughan-Davis model: sacrum between posterior superior iliac spines, anterior superior iliac spines, femoral greater trochanter, femoral lateral epicondyle, the head of fibula, lateral malleolus, calcaneal tuber, the head of the fifth metatarsal and markers on the bar on the lateral side of the thigh and shank (Davis et al., 1991; Vaughan, Davis \& O'Connor, 1992) (Fig. 1).

A short dynamic stretching preceded the set of two series of free body weight squat (FBWS) with hands in front (ten repetitions each). If no complaints were reported due to measuring instrumentations or any other cause, the third series of FBWS (seven repetitions) was recorded and then used to normalize the EMG signal. The mean EMG taken from three middle repetitions of FBWS for each muscle was used as a squat reference value (SRV). The main part of the experiment began with the HBBS technique. Each participant performed a short warm up squat series gradually increasing barbell weight, then proceeded to the nine HBBS testing trials $(3 \mathrm{x}$ $60 \%, 3 \times 65 \%$ and $3 \times 70 \%$ of subject's $1 \mathrm{RM})$. The same procedure was performed for LBBS technique after 30 minutes rest. All squats were visually inspected to confirm the proper technique and depth (thigh parallel with the floor or lower) (Aspe \& Swinton, 2014; Technical Rules Book, 2019).

The squat cycle (SC), its eccentric and concentric phases, depth, anterior pelvis tilt (PT), hip (HFE), knee (KFE) and ankle (AFE) joint angles (on the sagittal plane) were determined with the use of a motion capture optoelectronic system BTS Smart-D $200 \mathrm{~Hz}$ (BTS Bioengineering, Milan, Italy). The eccentric phase of the SC started from the highest vertical position of the marker (set on the sacrum bone between posterior superior iliac spines) and ended in its lowest vertical position. The concentric phase was defined respectively from the lowest position to the highest. The set of FBWS was used for the EMG data normalization. All tests were performed at a self-selected cadence.

\section{Instrumentation}

A Telemyo 2400T G2 device (Noraxon, USA) integrated and synchronized with optoelectronic system BTS Smart-D (BTS Bioengineering, Milan, Italy) was used to record surface EMG activity. The EMG signal was sampled at $800 \mathrm{~Hz}$ and then bandpass filtered (bandwidth: 10-400 $\mathrm{Hz}$. EMG signal processing was performed with MyoResearch XP Master Edition software (Noraxon, USA). Artefacts and noise were visually inspected. The EMG signal was full-wave rectified and smoothed using root mean square algorithm (RMS) with 50-ms windows. The peak and mean EMG values during the eccentric and concentric phases of HBBS and LBBS were calculated and presented in \%SRV.

Squat depth, PT, HFE, KFE and AFE for the lowest position of the sacrum marker together with the SC temporal characteristics were calculated in Smart Analyzer (BTS Bioengineering, Milan, Italy) using Euler angles convention (Fig. 2) (Davis et al., 1991).

Peer) reviewing PDF | (2020:01:45331:2:0:NEW 5 May 2020) 
159

160

161

162

163

164

165

166

167

168

169

170

171

172

173

174

175

176

177

178

179

180

181

182

183

184

185

186

187

188

189

190

191

192

193

194

195

196

197

198

\section{Statistical analyses}

Intraclass correlation coefficients (ICCs) for the independent variables from the 3 trials to determine test-retest reliability were calculated (95\% confidence interval). ICCs were considered as being poor (less than 0.5), moderate (between 0.5 and 0.75), good (between 0.75 and 0.9 ) and excellent (greater than 0.9) (Koo \& $\mathrm{Li}, 2016$ ). Repeated measures analysis of variance (ANOVA) was performed for angles $(2 \times 2 \times 3$, technique $[\mathrm{HB}$ or $\mathrm{LB}] \times \mathrm{LE}$ [left or right] $\times 1 \mathrm{RM}[60 \%$, $65 \%$ or $70 \%$ ] ) as well as for bioelectrical activity $(2 \times 2 \times 2 \times 3$, technique [HB or LB] $\times$ LE [left or right] $\times$ contraction [eccentric or concentric] $\times 1 \mathrm{RM}[60 \%, 65 \%$ or $70 \%])$. A Bonferroni adjustment was used to examine differences between within-subject factors. Sphericity was evaluated using the Mauchly test and Geisser-Greenhouse adjustments were made when sphericity was violated. Effect size estimates for the ANOVA test were determined by the partial eta-squared $\left(\eta^{2}\right) ; \eta^{2}$ values were interpreted according to the Cohen guidelines of small (0.01), medium (0.06) and large (0.14) (Cohen, 1988). For all analyses, the alpha level was set at $p<0.05$. All statistical analyses were performed in SPSS Statistics software for Windows (version 24.0, IBM Corp, Armonk, NY, USA).

\section{Results}

The mean \pm standard deviation (SD) of angles and depth for HBBS and LBBS at the 60\% 1RM, $65 \% 1 \mathrm{RM}$ and 70\% 1RM are presented in the Fig. 2. Moreover, Fig. 3 illustrates the mean values of the bioelectrical activity for HBBS and LBBS at the 60\% 1RM, 65\% 1RM and 70\% 1RM during the eccentric and concentric phases.

\section{Kinematics}

The ICCs values for the mean of 3 trials for the angles ranged from 0.89 to $0.99(p<0.001)$ indicated good and excellent reliability. Considering the technique factor, the analysis of the main effect demonstrated significant differences in the values of AFE $\left(\mathrm{F}_{1,30}=28.19\right.$; $\left.\eta^{2}=3302.56 ; p<0.001\right), \operatorname{KFE}\left(\mathrm{F}_{1,30}=33.89 ; \eta^{2}=2878.52 ; p<0.001\right), \operatorname{HFE}\left(\mathrm{F}_{1,30}=56.65\right.$ $\left.\eta^{2}=859.96 ; p<0.001\right)$ and PT $\left(\mathrm{F}_{1,30}=24.78 ; \eta^{2}=545.32 ; p<0.001\right)$. In the case of 1RM factor, was found significant main effect for $\operatorname{KFE}\left(\mathrm{F}_{2,60}=33.3 ; \eta^{2}=18.66 ; p=0.043\right)$ and $\mathrm{HFE}$ $\left(\mathrm{F}_{2,60}=4.29 ; \eta^{2}=6.50 ; p=0.018\right)$. In addition, was noted significant interaction effect between the technique and $1 \mathrm{RM}$ factors for $\operatorname{AFE}\left(\mathrm{F}_{2,60}=6.60 ; \eta^{2}=16.50 ; p=0.003\right), \operatorname{KFE}\left(\mathrm{F}_{2,60}=3.78\right.$; $\left.\eta^{2}=20.46 ; p=0.029\right)$ and $\operatorname{HFE}\left(\mathrm{F}_{2,60}=3.30 ; \eta^{2}=4.85 ; p=0.044\right)$. Overall, angles displayed a large effect size.

The percentage differences in the angle values between the HBBS and LBBS, and $p$-values of post-hoc test are shown in Table 1. Pairwise comparisons indicated significantly higher values of (1) AFE and KFE for HBBS than LBBS, and (2) PT for LBBS than HBBS $(p<0.001)$. Analysis showed also no significant differences between the 60\% 1RM, 65\% 1RM and 70\% 1RM $(p>0.05)$ as well as between the left and right lower extremities $(p>0.05)$ for all angles. 


\section{EMG}

200 The ICCs values for the mean of 3 trials for the bioelectrical activity ranged from 0.87 to 0.99

201 $(p<0.001)$ indicated good and excellent reliability. The analysis revealed significant main effect

202 of the technique factor for LES $\left(\mathrm{F}_{1,28}=74.13 ; \eta^{2}=19.77 ; p<0.001\right), \mathrm{GM}\left(\mathrm{F}_{1,28}=77.46\right.$;

$\left.203 \eta^{2}=49.49 ; p<0.001\right), \operatorname{BF}\left(\mathrm{F}_{1,28}=75.00 ; \eta^{2}=62.11 ; p<0.001\right)$ and $\operatorname{VLO}\left(\mathrm{F}_{1,28}=12.42\right.$;

$\left.204 \eta^{2}=0.89 ; p=0.001\right)$. Significant main effect was also observed for 1RM factor for LES

$205 \quad\left(\mathrm{~F}_{2,56}=91.80 ; \eta^{2}=4.65 ; p<0.001\right), \mathrm{GM}\left(\mathrm{F}_{2,56}=102.73 ; \eta^{2}=34.68 ; p<0.001\right), \mathrm{BF}$

$206 \quad\left(\mathrm{~F}_{2,56}=46.00 ; \eta^{2}=7.44 ; p<0.001\right), \mathrm{RF}\left(\mathrm{F}_{2,56}=38.90 ; \eta^{2}=4.94 ; p<0.001\right), \mathrm{VLO}\left(\mathrm{F}_{2,56}=85.07\right.$;

$\left.207 \eta^{2}=2.59 ; p<0.001\right)$ and $\operatorname{VMO}\left(\mathrm{F}_{2,56}=190.96 ; \eta^{2}=1.01 ; p<0.001\right)$. Moreover, significant

208 interaction effect between the technique and $1 \mathrm{RM}$ factors for LES was found $\left(\mathrm{F}_{2,56}=4.36\right.$;

$\left.209 \eta^{2}=0.09 ; p=0.017\right), \operatorname{GM}\left(\mathrm{F}_{2,56}=23.51 ; \eta^{2}=1.63 ; p<0.001\right), \operatorname{VLO}\left(\mathrm{F}_{2,56}=6.11 ; \eta^{2}=0.58\right.$;

$210 p=0.004)$ and $\operatorname{VMO}\left(\mathrm{F}_{2,56}=10.50 ; \eta^{2}=0.05 ; p<0.001\right)$. Results of the $\eta^{2}$ for bioelectrical

211 activity showed generally large effect size. The percentage differences in the bioelectrical

212 activity values between the HBBS and LBBS, and $p$-values of post-hoc test are shown in

213 Table 1. Pairwise comparisons demonstrated significantly lower values (1) for HBBS than LBBS

214 for LES, GM and BF (all 1RM; eccentric and concentric), VLO (60\% 1RM and 65\% 1RM;

215 eccentric) and VMO (all 1RM; eccentric), (2) for LBBS than HBBS for RF (all 1RM; eccentric

216 and concentric), VLO (65\% 1RM and 70\% 1RM; concentric) and VMO (65\% 1RM; concentric)

$217(p<0.05)$. The percentage differences in the bioelectrical activity between the 60\% 1RM, 65\%

$2181 \mathrm{RM}$ and 70\% 1RM, and $p$-values of post-hoc test are presented in Table 2. Comparisons

219 showed significantly lower values (1) at the 60\% 1RM than 65\% 1RM for all muscles (HBBS;

220 eccentric and concentric), GM (LBBS; eccentric), and BF and RF (LBBS; eccentric and

221 concentric), (2) at the 65\% 1RM than 70\% 1RM for all muscles (HBBS and LBBS; eccentric),

222 LES (HBBS; concentric), GM (HBBS and LBBS; concentric), and VMO (LBBS; concentric),

223 (3) at the 60\% 1RM than 70\% 1RM for all muscles (HBBS and LBBS; eccentric and concentric)

$224(p<0.05)$. In addition, EMG results were significantly lower during eccentric contraction than 225 concentric contraction for all muscle groups $(p<0.001)$ (Table 3$)$. However for the side factor, 226 analysis demonstrated no significant differences in the bioelectrical activity values between the 227 left and right lower extremities $(p>0.05)$.

228

229 Discussion

230 To the authors knowledge this study is first to investigate muscle activity differences between the 231 HBBS and LBBS within homogeneous group during the single measuring session without 232 repositioning of EMG probes. This methodology was chosen since changes in orientation of 233 surface electrodes and muscle fibers are known to affect the EMG amplitude (Paoli, Marcolin \& 234 Petrone, 2009). In that, our approach differs from previous studies where the comparisons 235 between HBBS and LBBS were made among different groups of lifters (Wretenberg, Feng \& 236 Arborelius, 1996) or it did not even include EMG activity (Swinton et al., 2012; Glassbrook et $237 a l ., 2017)$. Another important novelty is that the EMG signal normalization was based on FBWS 238 tests. 
239 This study provides also notable comparisons between different barbell loads ( $60 \%$ and $65 \%$, $24065 \%$ and $70 \%$ as well as $60 \%$ and $70 \%$ of prior tested $1 \mathrm{RM}$ ). Given that squat involves a 241 symmetrical movement pattern and there were no significant differences in measured angles and 242 bioelectrical activity between left and right lower extremities, the analysis was run only on the 243 right side.

244 The main findings of this research are that the EMG bioelectrical activity during eccentric phase 245 of squat motion for all selected muscles were significantly higher during LBBS than in HBBS 246 (60\% 1RM and 65\% 1RM). During 70\% 1RM squat test, those differences were also significant, 247 except for RF and VLO. During the concentric phase, a significantly greater muscle activity was 248 observed during LBBS for LES, GM and BF for all tested loads. These findings indicate that posterior muscles of lower extremities - hip extensors, were considerably more activated during LBBS compared to HBBS. For knee extensors, such differences were negligible and during $60 \%$ 1RM insignificant. It should be noticed that for the eccentric phase of the squat, the GM and LES muscles activated the most (both over 200\% SRV during LBBS with 70\% 1RM). The biggest differences between muscles activity for HBBS and LBBS eccentric phase are also demonstrated by the same muscles (GM and LES). This is due to the lower position of the bar in LBBS, which imposes higher anterior PT (Fig. 2) and a more forward trunk position, together with the wider foot stance (McCaw \& Melrose, 1999; Paoli, Marcolin \& Petrone, 2009).

257 All examined muscles were more activated during concentric phase of the $\mathrm{SC}$ which perfectly corresponds with previous works (Selseth et al., 2000; Ebben \& Jensen, 2002; Gullett et al., 2009). Mean EMG activity recorded in that phase for each muscle clearly exceeds $200 \%$ SRV

260

261

262

263

264

265

266

267

268

269

270

271

272

273

274

275

276

277

278 (for each barbell load level). Similar to the eccentric phase, the most activated muscle during concentric phase was GM (almost 700\% SRV during LBBS with 70\% 1RM). GM muscle activates closely twice as much as the other recorded muscle, with the exception of BF which is also very well engaged ( $446 \% \mathrm{SRV})$. What is even more notable is that GM muscle during concentric phase is activated approximately three times more than during eccentric phase for the LBBS, and almost four times more for the HBBS. This may come from more upright position of the torso in HBBS than in LBBS, which was suggested as one of the reasons for hamstring active insufficiency (due to shortened hamstring muscle length in that position), and for the presence of compensation strategy observable by greater GM activity (Glassbrook et al., 2017).

Unlike the eccentric phase, the biggest differences between muscle activity for HBBS and LBBS concentric phases were observed at the BF level. This proves another crucial factor in deciding which type of squat is more desirable at the moment. The reason for that may be explained by the BF stretch-shortening SC, because the wider foot stance, more forward torso position and bigger anterior PT in LBBS stretches BF more (Gordon, Huxley \& Julian, 1966; Escamilla et al., 2001).

The additional goal of this study was to verify the influence of load level on the EMG activity for HBBS and LBBS. Although the load level effect on EMG activity is well described in previous studies (McCaw \& Friday, 1994; McCaw \& Melrose, 1999), the difference characteristics between HBBS and LBBS was still not known. 
279 It is interesting that in the concentric phase significant differences between the loads are 280 generally not observed between just 5\% 1RM change in load level for LBBS, while for HBBS 281 they are noticeable only between $60 \% 1 \mathrm{RM}$ and $65 \% 1 \mathrm{RM}$. As we observed much bigger 282 activity values for all muscles for concentric phase, this observation may indicate that for the 283 more significant progress in LBBS training it is advisable to progress the load with at least $10 \%$ 284 1RM (significant differences observed for both HBBS and LBBS). This observation only applies 285 to the concentric phase of the $\mathrm{SC}$ where muscle activity level is the highest. In the eccentric 286 phase significant differences emerged between all analyzed loads with the exception of $60 \%$ to $28765 \%$ 1RM comparison for LES, VLO and VMO. Load progress affects most effectively GM and 288 BF during eccentric phase of HBBS while the least significant influence can be observed for 289 VLO and VMO during LBBS (concentric).

290 This study clearly pointed significant differences in EMG muscle activity between HBBS and

291

292

293

294

295

296

297

298

299

300

301

302

303

304

305

306

307

308

309

310

311

312

313

314

315

316

317

318

LBBS. It appears that crucial differences occur for the hip extensors (GM, BF) and LES muscles. The significant differences in the EMG activity between superficially similar HBBS and LBBS is even more interesting when these results are compared with the work of Gullett et al. (2009), who found no differences between totally different bar position squat techniques - front and back squat (for six muscles, unfortunately without GM). Other squat variations with variable resistance, like elastic bands or chains attached to the bar, did not provide sufficient difference in muscle activation (Ebben \& Jensen, 2002; Gullett et al., 2009; Saeterbakken, Andersen \& van den Tillaar, 2016). For the knee extensors muscles, the difference between HBBS and LBBS is not that obvious. The differences are rather negligible which corresponds to some previous works (McCaw \& Melrose \& 1999; Clark, Lambert \& Hunter, 2012). McCaw \& Melrose (1999) also analyzed the activity of RF, VLO and VMO. They focused on the effect of different stance width during the parallel LBBS, but they found no change in quadriceps EMG activity either (McCaw \& Melrose, 1999; Clark, Lambert \& Hunter, 2012). Clark, Lambert \& Hunter (2012) indicated the same muscle length, as the cause of no significant differences in quadriceps EMG activity, between the various types of width stance. Because of very small differences in KFE, this argumentation may also explain minor differences for RF, VLO and VMO between HBBS and LBBS.

The potential limitation of this study was that most of the athletes were preparing for the National Academic Championships, so in order not to interfere with individual training preparations (Issurin, 2010), the load level was limited to 70\% of 1RM. Such load level also let the athletes perform their squats with the optimum and repeatable technique, which was designed to make the comparisons between both techniques more reliable. In future studies it is worth to use similar procedure and analyse differences between HBBS and LBBS during 90\% of 1RM to $100 \%$ of $1 \mathrm{RM}$ performances.

\section{Conclusions}

This study is the first to compare HBBS and LBBS on the homogeneous group of experienced powerlifters. Our results confirmed the significant differences in posterior muscle chain

Peer) reviewing PDF | (2020:01:45331:2:0:NEW 5 May 2020) 
319 activation between both squat techniques. LES, GM, BF, RF, VLO and VMO activity during 320 eccentric phase of squat motion were significantly higher during LBBS than HBBS. For the knee 321 extensors muscles, the difference between HBBS and LBBS are rather negligible. GM and BF 322 muscles are the most crucial from tested muscles for both HBBS and LBBS but it is the LBBS 323 which engages the muscles at the highest level. The outcomes may be useful in designing 324 specific training programs and in optimizing performance. Our findings expand the actual 325 knowledge providing quantitative muscular activation data. The lower bar position and the wider 326 foot stance may remarkably influence the final result of athletes competing in powerlifting 327 competition.

328

\section{Acknowledgements}

330

331

332

333

334

335

336

337

338

339

340

341

342

343

344

345

346

347

348

349

350

351

352

353

354

355

356

357

The authors would like to thank all participating athletes. The authors declare that they have no conflict of interests.

\section{References}

Ahankoob N, Chokshi M, Feinstein M, Stone NJ. 2011. Should the routine physical examination include squat maneuvers? American Journal of Medicine 124:702-704

DOI: 10.1016/j.amjmed.2011.01.024.

Aspe RR, Swinton PA. 2014. Electromyographic and kinetic comparison of the back squat and overhead squat. Journal of Strength and Conditioning Research 28:2827-2836 DOI: $10.1519 / \mathrm{JSC} .0000000000000462$.

Banyard HG, Nosaka K, Sato K, Haff GG. 2017. Validity of various methods for determining velocity, force, and power in the back squat. International Journal of Sports Physiology and Performance 12:1170-1176 DOI: 10.1123/ijspp.2016-0627.

Bryanton MA, Kennedy MD, Carey JP, Chiu LZF. 2012. Effect of squat depth and barbell load on relative muscular effort in squatting. Journal of Strength and Conditioning Research 26:2820-2828 DOI: 10.1519/JSC.0b013e31826791a7.

Clark D, Lambert MI, Hunter AM. 2016. Reliability of trunk muscle electromyography in the loaded back squat exercise. International Journal of Sports Medicine 37:448-456

DOI: $10.1055 / \mathrm{s}-0035-1569366$.

Clark DR, Lambert MI, Hunter AM. 2012. Muscle activation in the loaded free barbell squat: A brief review. Journal of Strength and Conditioning Research 26:1169-1178

DOI: $10.1519 / \mathrm{JSC} .0 \mathrm{~b} 013 \mathrm{e} 31822 \mathrm{~d} 533 \mathrm{~d}$.

Cohen J. 1988. Statistical power analysis for the behavioral sciences. Hillsdale, NJ: Lawrence Erlbaum Associates.

Contreras B, Vigotsky AD, Schoenfeld BJ, Beardsley C, Cronin J. 2015. A comparison of gluteus maximus, biceps femoris, and vastus lateralis electromyographic activity in the back squat and barbell hip thrust exercises. Journal of Applied Biomechanics 31:452-458

DOI: $10.1123 / \mathrm{jab} .2014-0301$.

Peer) reviewing PDF | (2020:01:45331:2:0:NEW 5 May 2020) 
358 Contreras B, Vigotsky AD, Schoenfeld BJ, Beardsley C, Cronin J. 2016. A comparison of

359

360

361

362

363

364

365

366

367

368

369

370

371

372

373

374

375

376

377

378

379

380

381

382

383

384

385

386

387

388

389

390

391

392

393

394

395

396

397

gluteus maximus, biceps femoris, and vastus lateralis electromyography amplitude in the parallel, full, and front squat variations in resistance-trained females. Journal of Applied Biomechanics 32:16-22 DOI: 10.1123/jab.2015-0113.

Davis RB, Ounpuu S, Tyburski D, Gage JR. 1991. A gait analysis data collection and reduction technique. Human Movement Science 10:575-587 DOI: https://doi.org/10.1016/01679457(91)90046-Z.

Earp JE, Newton RU, Cormie P, Blazevich AJ. 2016. Faster movement speed results in greater tendon strain during the loaded squat exercise. Frontiers in Physiology 7:366

DOI: $10.3389 /$ fphys.2016.00366.

Ebben WP, Jensen RL. 2002. Electromyographic and kinetic analysis of traditional, chain, and elastic band squats. Journal of Strength and Conditioning Research 16:547-550.

Escamilla RF, Fleisig GS, Lowry TM, Barrentine SW, Andrews JR. 2001. A three-dimensional biomechanical analysis of the squat during varying stance widths. Medicine and Science in Sports and Exercise 33:984-998. DOI: 10.1097/00005768-200106000-00019.

Fujita E, Kanehisa H, Yoshitake Y, Fukunaga T, Nishizono H. 2011. Association between knee extensor strength and EMG activities during squat movement. Medicine and Science in Sports and Exercise 43:2328-2334 DOI: 10.1249/MSS.0b013e3182207ed8.

Glassbrook DJ, Helms ER, Brow, SR, Storey AG. 2017. A review of the biomechanical differences between the high-bar and low-bar back-squat. Journal of Strength and Conditioning Research 31:2618-2634 DOI: 10.1519/JSC.0000000000002007.

Glassbrook DJ, Brown SR, Helms ER, Duncan JS, Storey AG. 2019. The high-bar and low-bar back-squats: A biomechanical analysis. Journal of Strength and Conditioning Research 33:1-13 DOI: $10.1519 / \mathrm{JSC} .0000000000001836$.

Gordon A, Huxley AF, Julian F. 1966. The variation in isometric tension with sarcomere length in vertebrate muscle fibres. Journal of Physiology 184:170-192.

Gullett JC, Tillman MD, Gutierrez GM, Chow JW. 2009. A biomechanical comparison of back and front squats in healthy trained individuals. Journal of Strength and Conditioning Research 23:284-292 DOI: 10.1519/JSC.0b013e31818546bb.

Hammer RL, Linton JT, Hammer AM. 2018. Effects of heavy squat training on a vibration platform on maximal strength and jump performance in resistance-trained men. Journal of Strength and Conditioning Research 32:1809-1815 DOI: 10.1519/JSC.0000000000002565. Hammond A, Perrin C, Steele J, Giessing J, Gentil P, Fisher JP. 2019. The effects of a 4-week mesocycle of barbell back squat or barbell hip thrust strength training upon isolated lumbar extension strength. PeerJ 7:e7337. DOI: 10.7717/peerj.7337.

Hermens HJ, Freriks B, Disselhorst-Klug C, Rau G. 2000. Development of recommendations for SEMG sensors and sensor placement procedures. Journal of Electromyography and Kinesiology 10:361-374 DOI: 10.1016/s1050-6411(00)00027-4.

Issurin VB. 2010. New horizons for the methodology and physiology of training periodization. Sports Medicine 40:189-206 DOI: 10.2165/11319770-000000000-00000.

Peer) reviewing PDF | (2020:01:45331:2:0:NEW 5 May 2020) 
398 Kasman GS, Cram JR, Wolf SL, Barton L. 1998. Clinical applications in surface

399 electromyography for chronic musculosceletal pain. Gaithersburg, Maryland: Aspen Publishers.

400 Koo TK, Li MY. 2016. A guideline of selecting and reporting intraclass correlations coefficients

401 for reliability research. Journal of Chiropractic medicine 15:155-163

402 DOI: $10.1016 / \mathrm{j} . \mathrm{jcm} .2016 .02 .012$.

403 Kritz M, Cronin J, Hume P. 2009. The bodyweight squat: A movement screen for the squat 404 pattern. Strength and Conditioning Journal 31:76-85 DOI: 10.1519/SSC.0b013e318195eb2f.

405 Kushner AM, Brent JL, Schoenfeld BJ, Hugentobler J, Lloyd RS, Vermeil A, Chu DA, Harbin J, 406 McGill SM, Myer GD. 2015. The back squat: Targeted training techniques to correct functional 407 deficits and technical factors that limit performance. Strength and Conditioning Journal 37:1340860 DOI: $10.1519 /$ SSC.0000000000000130.

409 McBride JM, Skinner JW, Schafer PC, Haines TL, Kirby TJ. 2010. Comparison of kinetic 410 variables and muscle activity during a squat vs. a box squat. Journal of Strength and 411 Conditioning Research 24:3195-3199 DOI: 10.1519/jsc.0b013e3181f6399a.

412 McCaw ST, Friday JJ. 1994. A comparison of muscle activity between a free weight and 413 machine bench press. Journal of Strength and Conditioning Research 8:259-264

414 DOI: 10.1519/JSC.0b013e3181cc2237.

415 McCaw ST, Melrose DR. 1999. Stance width and bar load effects on leg muscle activity during 416 the parallel squat. Medicine and Science in Sports Exercise 31:428-436

417 DOI: 10.1097/00005768-199903000-00012.

418 Myer GD, Kushner AM, Brent JL, Schoenfeld BJ, Hugentobler J, Lloyd RS, Vermeil A, Chu 419 DA, Harbin J, McGill SM. 2014. The back squat: A proposed assessment of functional deficits 420 and technical factors that limit performance. Strength and Conditioning Journal 36:4-27 421 DOI: $10.1519 / \mathrm{SSC} .0000000000000103$.

422 Niewiadomski W, Laskowska D, Gąsiorowska A, Cybulski G, Strasz A, Langfort J. 2008. 423 Determination and prediction of one repetition maximum (1RM): Safety considerations. Journal 424 of Human Kinetics 19:109-120 DOI: 10.2478/v10078-008-0008-8.

425 Paoli A, Marcolin G, Petrone N. 2009. The effect of stance width on the electromyographical 426 activity of eight superficial thigh muscles during back squat with different bar loads. Journal of 427 Strength and Conditioning Research 23:246-250 DOI: 10.1519/jsc.0b013e3181876811.

428 Rabin A, Kozol Z. 2017. Utility of the overhead squat and forward arm squat in screening for 429 limited ankle dorsiflexion. Journal of Strength and Conditioning Research 31:1251-1258 430 DOI: 10.1519/JSC.0000000000001580.

431 Saeterbakken AH, Andersen V, van den Tillaar R. 2016. Comparison of kinematics and muscle 432 activation in free-weight back squat with and without elastic bands. Journal of Strength and 433 Conditioning Research 30:945-952 DOI: 10.1519/JSC.0000000000001178.

434 Schwanbeck S, Chilibeck PD, Binsted G. 2009. A comparison of free weight squat to smith 435 machine squat using electromyography. Journal of Strength and Conditioning Research 436 23:2588-2591 DOI: 10.1519/JSC.0b013e3181b1b181. 
437 Selseth A, Dayton M, Cordova M, Ingersoll CD, Merrick MA. 2000. Quadriceps concentric 438 EMG activity is greater than eccentric EMG activity during the lateral step-up exercise. Journal 439 of Sport Rehabilitation 9:124-134 DOI: https://doi.org/10.1123/jsr.9.2.124.

440 Shariat A, Lam ETC, Shaw BS, Shaw I, Kargarfard M, Sangelaji B. 2017. Impact of back squat 441 training intensity on strength and flexibility of hamstring muscle group. Journal of Back and 442 Musculoskeletal Rehabilitation 30:641-647 DOI: 10.3233/BMR-160526.

443 Swinton PA, Lloyd R, Keogh JWL, Agouris I, Stewart AD. 2012. A biomechanical comparison 444 of the traditional squat, powerlifting squat, and box squat. Journal of Strength and Conditioning 445 Research 26:1805-1816 DOI: 10.1519/JSC.0b013e3182577067.

446 Technical Rules Book. 2019. International Powerlifting Federation. Available at 447 https://www.powerlifting.sport/rulescodesinfo/technical-rules.html.

448 Usui S, Maeo S, Tayashiki K, Nakatani M, Kanehisa H. 2016. Low-load slow movement squat 449 training increases muscle size and strength but not power. International Journal of Sports 450 Medicine 37:305-312 DOI: 10.1055/s-0035-1564255.

451 Vaughan C, Davis B, O’Connor J. 1992. Dynamics of human gait. Cape Town: Kiboho 452 Publishers.

453 Wirth K, Hartmann H, Sander A, Mickel C, Szilvas E, Keiner M. 2016. The impact of back squat 454 and leg-press exercises on maximal strength and speed-strength parameters. Journal of Strength 455 and Conditioning Research 30:1205-1212 DOI: 10.1519/JSC.0000000000001228.

456 Wisloff U, Castagna C, Helgerud J, Jones R, Hoff J. 2004. Strong correlation of maximal squat 457 strength with sprint performance and vertical jump height in elite soccer players. British Journal 458 of Sports Medicine 38:285-288 DOI: 10.1136/bjsm.2002.002071.

459 Wretenberg P, Feng Y, Arborelius UP. 1996. High- and low-bar squatting techniques during 460 weight-training. Medicine and Science in Sports and Exercise 28:218-224

461 DOI: 10.1097/00005768-199602000-00010.

462 
Figure 1

Placement of reflective markers ( $A$, anterior; $B$, posterior).

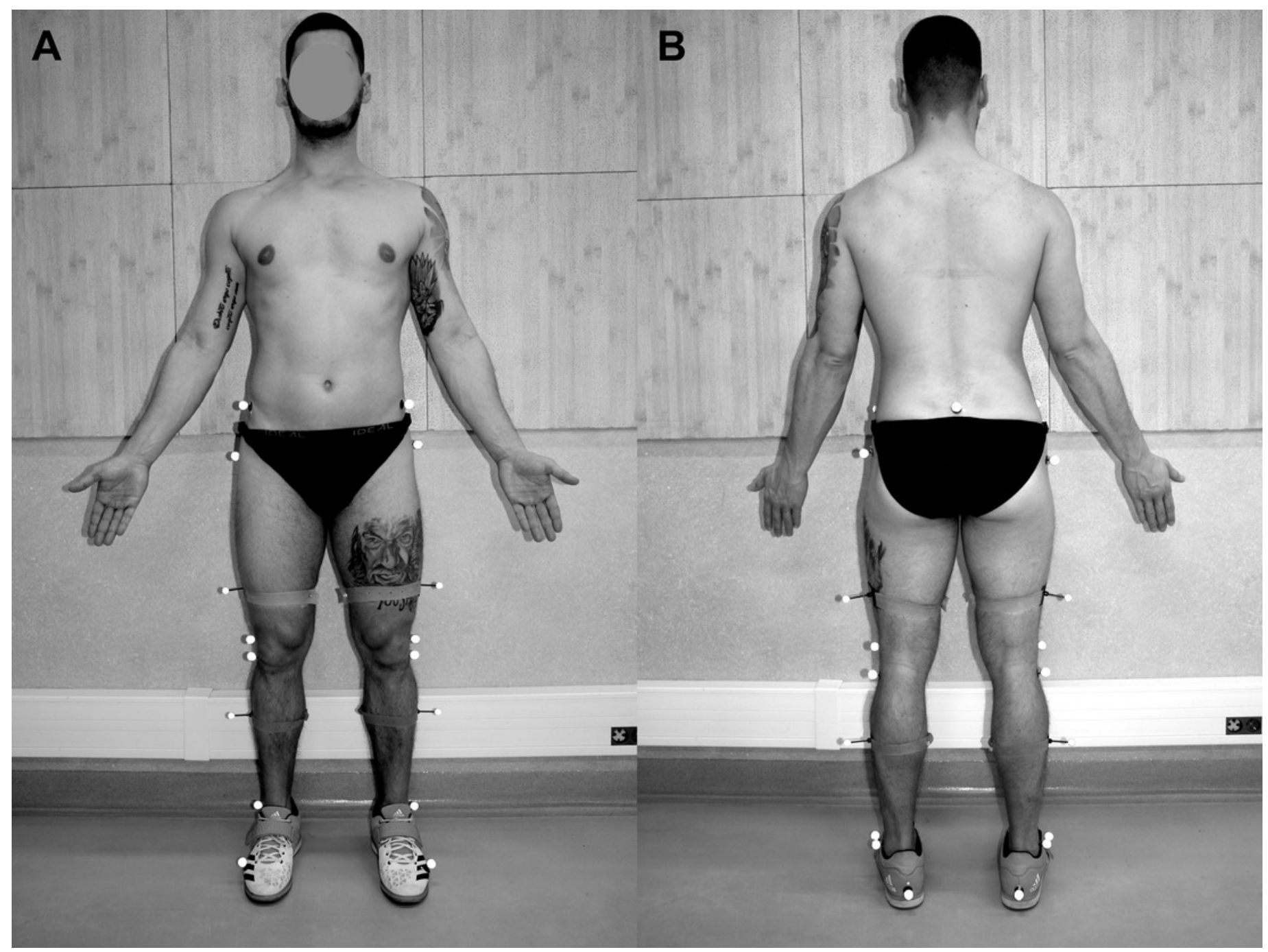


Figure 2

Mean \pm SD of the PT, HFE, KFE, AFE and depth (A, for HBBS; B, for LBBS).

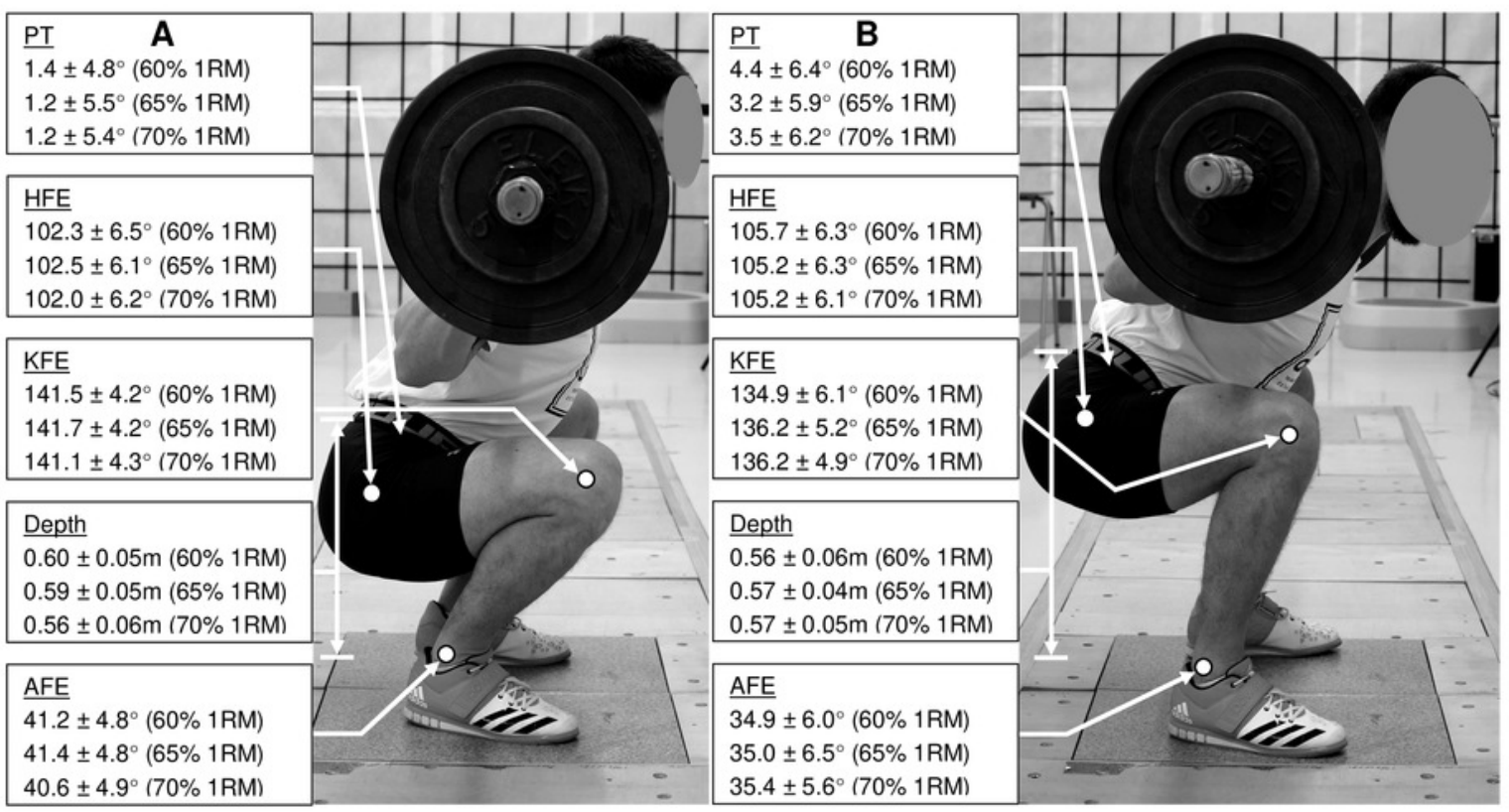


Figure 3

Bioelectrical activity values of the LES, GM, BF, RF, VLO and VMO for HBBS and LBBS (A, eccentric phase; B, concentric phase).
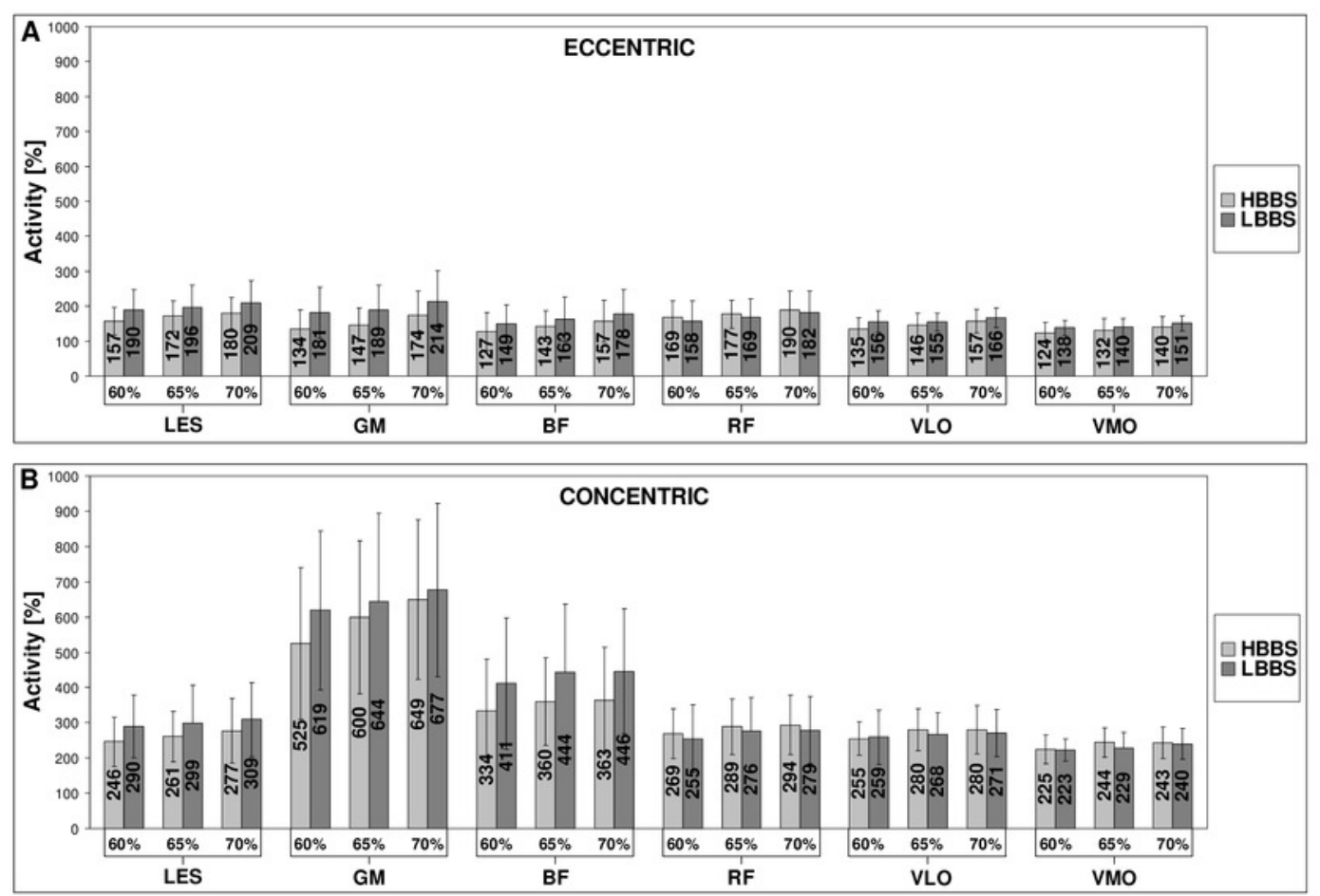


\section{Table $\mathbf{1}$ (on next page)}

Angle and activity differences between the HBBS and LBBS for $60 \%, 65 \%$ and $70 \% 1$ RM (mean \pm SD) and $p$-values.

HBBS, high-bar back squat; LBBS, low-bar back squat; 1RM, one repetition maximum; SD, standard deviation; Diff, difference; AFE, ankle flexion extension angle; KFE, knee flexion extension angle; HFE, hip flexion extension angle; PT, pelvis tilt angle; LES, lumbar erector spinae; GM, gluteus maximus; BF, biceps femoris; RF, rectus femoris; VLO, vastus lateralis; VMO, vastus medialis; * significant differences $(p \leq 0.05)$ between the HBBS and HBBS. 


\begin{tabular}{|c|c|c|c|c|c|c|}
\hline \multirow{2}{*}{ Variable } & \multicolumn{2}{|c|}{$60 \% 1 \mathrm{RM}$} & \multicolumn{2}{|c|}{$65 \% 1 \mathrm{RM}$} & \multicolumn{2}{|c|}{$70 \% 1 \mathrm{RM}$} \\
\hline & $\operatorname{Diff}[\%]$ & $p$ & $\operatorname{Diff}[\%]$ & $p$ & $\operatorname{Diff}[\%]$ & $p$ \\
\hline \multicolumn{7}{|l|}{ Angle } \\
\hline $\mathrm{AFE}$ & $14.3 \pm 12.5^{*}$ & $<0.001$ & $14.7 \pm 11.3^{*}$ & $<0.001$ & $12.2 \pm 9.0^{*}$ & $<0.001$ \\
\hline KFE & $4.6 \pm 3.0^{*}$ & $<0.001$ & $3.8 \pm 2.7^{*}$ & $<0.001$ & $3.5 \pm 2.7^{*}$ & $<0.001$ \\
\hline HFE & $-3.2 \pm 2.4$ & 0.158 & $-2.5 \pm 1.6$ & 0.086 & $-3.0 \pm 1.6$ & 0.106 \\
\hline PT & $-30.6 \pm 20.6^{*}$ & $<0.001$ & $-26.4 \pm 18.3^{*}$ & $<0.001$ & $-33.3 \pm 12.6^{*}$ & $<0.001$ \\
\hline \multicolumn{7}{|c|}{ Activity, eccentric } \\
\hline LES & $-15.8 \pm 5.1^{*}$ & $<0.001$ & $-9.5 \pm 5.4^{*}$ & $<0.001$ & $-11.7 \pm 5.8^{*}$ & $<0.001$ \\
\hline GM & $-25.2 \pm 8.2 *$ & $<0.001$ & $-20.7 \pm 11.1^{*}$ & $<0.001$ & $-16.8 \pm 10.4^{*}$ & $<0.001$ \\
\hline $\mathrm{BF}$ & $-11.8 \pm 9.7^{*}$ & $<0.001$ & $-9.4 \pm 6.0^{*}$ & $<0.001$ & $-10.8 \pm 5.4^{*}$ & $<0.001$ \\
\hline $\mathrm{RF}$ & $8.4 \pm 4.9^{*}$ & 0.001 & $6.2 \pm 3.4^{*}$ & 0.024 & $5.3 \pm 4.3^{*}$ & 0.028 \\
\hline VLO & $-13.8 \pm 7.7^{*}$ & $<0.001$ & $-6.8 \pm 4.7^{*}$ & $<0.001$ & $-4.2 \pm 10.4$ & 0.372 \\
\hline VMO & $-11.2 \pm 8.0^{*}$ & $<0.001$ & $-6.7 \pm 5.6^{*}$ & $<0.001$ & $-8.7 \pm 5.9 *$ & $<0.001$ \\
\hline \multicolumn{7}{|c|}{ Activity, concentric } \\
\hline LES & $-14.1 \pm 6.6^{*}$ & $<0.001$ & $-9.7 \pm 7.3^{*}$ & $<0.001$ & $-9.9 \pm 6.2 *$ & $<0.001$ \\
\hline GM & $-15.8 \pm 10.9^{*}$ & 0.021 & $-6.3 \pm 4.7^{*}$ & 0.003 & $-4.2 \pm 3.0^{*}$ & 0.005 \\
\hline $\mathrm{BF}$ & $-17.9 \pm 8.5^{*}$ & $<0.001$ & $-14.1 \pm 10.0^{*}$ & $<0.001$ & $-17.3 \pm 7.4^{*}$ & $<0.001$ \\
\hline $\mathrm{RF}$ & $7.5 \pm 3.4^{*}$ & 0.043 & $6.1 \pm 4.7^{*}$ & 0.020 & $6.3 \pm 4.6^{*}$ & 0.001 \\
\hline VLO & $0.4 \pm 5.0$ & 0.607 & $4.5 \pm 3.4^{*}$ & $<0.001$ & $3.5 \pm 1.9^{*}$ & $<0.001$ \\
\hline VMO & $-0.2 \pm 4.9$ & 0.656 & $6.3 \pm 5.0^{*}$ & $<0.001$ & $1.0 \pm 2.6$ & 0.348 \\
\hline
\end{tabular}




\section{Table 2 (on next page)}

Activity differences between the $60 \%, 65 \%$ and $70 \%$ 1RM for HBBS and LBBS (mean \pm SD) and p-values.

HBBS, high-bar back squat; LBBS, low-bar back squat; 1RM, one repetition maximum; SD, standard deviation; Diff, difference; LES, lumbar erector spinae; GM, gluteus maximus; BF, biceps femoris; RF, rectus femoris; VLO, vastus lateralis; * significant differences $(p \leq 0.05)$ between the $60 \%, 65 \%$ and $70 \% 1 R M$. 


\begin{tabular}{|c|c|c|c|c|c|c|}
\hline \multirow{2}{*}{ Technique } & \multicolumn{2}{|c|}{60 vs. $65 \% 1 \mathrm{RM}$} & \multicolumn{2}{|c|}{65 vs. $70 \% 1 \mathrm{RM}$} & \multicolumn{2}{|c|}{60 vs. $70 \% 1 \mathrm{RM}$} \\
\hline & $\operatorname{Diff}[\%]$ & $p$ & $\operatorname{Diff}[\%]$ & $p$ & $\operatorname{Diff}[\%]$ & $p$ \\
\hline \multicolumn{7}{|c|}{ HBBS, eccentric } \\
\hline LES & $-8.4 \pm 4.3^{*}$ & $<0.001$ & $-4.3 \pm 3.5^{*}$ & $<0.001$ & $-12.4 \pm 4.4^{*}$ & $<0.001$ \\
\hline GM & $-10.7 \pm 9.3^{*}$ & $<0.001$ & $-14.0 \pm 7.5^{*}$ & $<0.001$ & $-23.7 \pm 7.5^{*}$ & $<0.001$ \\
\hline $\mathrm{BF}$ & $-12.6 \pm 7.5^{*}$ & $<0.001$ & $-7.5 \pm 4.1^{*}$ & 0.012 & $-19.7 \pm 8.3^{*}$ & $<0.001$ \\
\hline $\mathrm{RF}$ & $-5.5 \pm 4.7^{*}$ & 0.002 & $-4.9 \pm 3.7^{*}$ & 0.003 & $-10.1 \pm 6.0^{*}$ & $<0.001$ \\
\hline VLO & $-7.4 \pm 5.4^{*}$ & $<0.001$ & $-7.1 \pm 5.3^{*}$ & $<0.001$ & $-14.1 \pm 4.9^{*}$ & $<0.001$ \\
\hline VMO & $-5.9 \pm 4.5^{*}$ & $<0.001$ & $-5.8 \pm 4.7^{*}$ & $<0.001$ & $-11.5 \pm 3.8^{*}$ & $<0.001$ \\
\hline \multicolumn{7}{|c|}{ HBBS, concentric } \\
\hline LES & $-5.5 \pm 3.3^{*}$ & 0.001 & $-3.9 \pm 2.4^{*}$ & 0.050 & $-9.4 \pm 7.0^{*}$ & $<0.001$ \\
\hline GM & $-13.2 \pm 5.8^{*}$ & $<0.001$ & $-7.4 \pm 4.1^{*}$ & $<0.001$ & $-19.8 \pm 8.5^{*}$ & $<0.001$ \\
\hline $\mathrm{BF}$ & $-10.4 \pm 8.6^{*}$ & $<0.001$ & $1.0 \pm 3.3$ & 0.998 & $-9.7 \pm 6.3^{*}$ & $<0.001$ \\
\hline $\mathrm{RF}$ & $-6.4 \pm 3.1^{*}$ & $<0.001$ & $-1.5 \pm 4.4$ & 0.428 & $-7.9 \pm 4.2^{*}$ & $<0.001$ \\
\hline VLO & $-8.5 \pm 5.5^{*}$ & $<0.001$ & $0.8 \pm 2.4$ & 0.996 & $-7.6 \pm 4.0^{*}$ & $<0.001$ \\
\hline VMO & $-7.9 \pm 4.4^{*}$ & $<0.001$ & $0.7 \pm 1.8$ & 1.000 & $-7.3 \pm 3.5^{*}$ & $<0.001$ \\
\hline \multicolumn{7}{|c|}{ LBBS, eccentric } \\
\hline LES & $-1.7 \pm 3.1$ & 0.064 & $-6.5 \pm 4.9^{*}$ & $<0.001$ & $-8.1 \pm 5.8^{*}$ & $<0.001$ \\
\hline GM & $-5.9 \pm 5.1^{*}$ & 0.021 & $-9.5 \pm 5.3^{*}$ & $<0.001$ & $-15.2 \pm 8.4^{*}$ & $<0.001$ \\
\hline $\mathrm{BF}$ & $-10.4 \pm 6.4^{*}$ & $<0.001$ & $-8.8 \pm 5.1^{*}$ & $<0.001$ & $-17.9 \pm 12.4^{*}$ & $<0.001$ \\
\hline $\mathrm{RF}$ & $-7.2 \pm 3.1^{*}$ & 0.008 & $-6.6 \pm 4.6^{*}$ & 0.001 & $-13.5 \pm 5.4^{*}$ & $<0.001$ \\
\hline VLO & $-0.0 \pm 2.4$ & 1.000 & $-5.2 \pm 4.6^{*}$ & $<0.001$ & $-5.0 \pm 5.0^{*}$ & $<0.001$ \\
\hline VMO & $-1.2 \pm 1.7$ & 0.132 & $-8.0 \pm 4.9^{*}$ & $<0.001$ & $-9.0 \pm 5.3^{*}$ & $<0.001$ \\
\hline \multicolumn{7}{|c|}{ LBBS, concentric } \\
\hline LES & $-1.2 \pm 4.7$ & 0.273 & $-3.7 \pm 3.3$ & 0.072 & $-5.0 \pm 3.8^{*}$ & 0.001 \\
\hline GM & $-3.3 \pm 3.1$ & 0.061 & $-5.2 \pm 4.3^{*}$ & 0.003 & $-8.6 \pm 5.8^{*}$ & $<0.001$ \\
\hline $\mathrm{BF}$ & $-7.0 \pm 5.8^{*}$ & $<0.001$ & $-1.9 \pm 4.3$ & 0.997 & $-8.9 \pm 5.7^{*}$ & $<0.001$ \\
\hline $\mathrm{RF}$ & $-7.7 \pm 3.8^{*}$ & 0.001 & $-1.5 \pm 2.4$ & 0.647 & $-9.1 \pm 5.8^{*}$ & $<0.001$ \\
\hline VLO & $-4.3 \pm 4.0$ & 0.059 & $-0.8 \pm 2.5$ & 0.516 & $-5.1 \pm 3.4^{*}$ & 0.001 \\
\hline VMO & $-1.5 \pm 3.5$ & 0.267 & $-4.6 \pm 3.7^{*}$ & $<0.001$ & $-6.0 \pm 3.7^{*}$ & $<0.001$ \\
\hline
\end{tabular}




\section{Table 3 (on next page)}

Activity differences between the eccentric and concentric contractions for HBBS and LBBS (mean $\pm S D$ ) and $p$-values.

HBBS, high-bar back squat; LBBS, low-bar back squat; 1RM, one repetition maximum; SD, standard deviation; Diff, difference; LES, lumbar erector spinae; GM, gluteus maximus; BF, biceps femoris; RF, rectus femoris; VLO, vastus lateralis; * significant differences $(p \leq 0.05)$ between the eccentric and concentric contractions. 


\begin{tabular}{|c|c|c|c|c|c|c|}
\hline \multirow{2}{*}{ Technique } & \multicolumn{2}{|c|}{$60 \% 1 \mathrm{RM}$} & \multicolumn{2}{|c|}{$65 \% 1 \mathrm{RM}$} & \multicolumn{2}{|c|}{$70 \% 1 \mathrm{RM}$} \\
\hline & $\operatorname{Diff}[\%]$ & $p$ & $\operatorname{Diff}[\%]$ & $p$ & $\operatorname{Diff}[\%]$ & $p$ \\
\hline \multicolumn{7}{|l|}{ HBBS } \\
\hline LES & $-34.8 \pm 7.7^{*}$ & $<0.001$ & $-33.1 \pm 4.5^{*}$ & $<0.001$ & $-32.7 \pm 7.6^{*}$ & $<0.001$ \\
\hline GM & $-74.5 \pm 5.1^{*}$ & $<0.001$ & $-75.4 \pm 3.2 *$ & $<0.001$ & $-73.5 \pm 3.7^{*}$ & $<0.001$ \\
\hline $\mathrm{BF}$ & $-61.6 \pm 3.4^{*}$ & $<0.001$ & $-60.5 \pm 4.7^{*}$ & $<0.001$ & $-56.8 \pm 3.8^{*}$ & $<0.001$ \\
\hline $\mathrm{RF}$ & $-37.3 \pm 3.0 *$ & $<0.001$ & $-37.7 \pm 5.5^{*}$ & $<0.001$ & $-35.4 \pm 4.7^{*}$ & $<0.001$ \\
\hline VLO & $-47.5 \pm 3.9^{*}$ & $<0.001$ & $-48.1 \pm 3.3^{*}$ & $<0.001$ & $-43.6 \pm 3.6^{*}$ & $<0.001$ \\
\hline VMO & $-45.5 \pm 3.6^{*}$ & $<0.001$ & $-46.5 \pm 5.0^{*}$ & $<0.001$ & $-42.9 \pm 3.2 *$ & $<0.001$ \\
\hline \multicolumn{7}{|l|}{ LBBS } \\
\hline LES & $-33.5 \pm 8.0 *$ & $<0.001$ & $-33.1 \pm 7.8^{*}$ & $<0.001$ & $-31.4 \pm 6.9 *$ & $<0.001$ \\
\hline GM & $-71.6 \pm 4.8 *$ & $<0.001$ & $-70.7 \pm 5.3^{*}$ & $<0.001$ & $-69.3 \pm 5.1 *$ & $<0.001$ \\
\hline $\mathrm{BF}$ & $-64.1 \pm 4.2 *$ & $<0.001$ & $-62.8 \pm 3.3 *$ & $<0.001$ & $-60.0 \pm 3.1 *$ & $<0.001$ \\
\hline $\mathrm{RF}$ & $-37.8 \pm 4.8^{*}$ & $<0.001$ & $-38.1 \pm 6.1 *$ & $<0.001$ & $-34.8 \pm 5.1^{*}$ & $<0.001$ \\
\hline VLO & $-38.6 \pm 5.9 *$ & $<0.001$ & $-41.4 \pm 4.0^{*}$ & $<0.001$ & $-35.1 \pm 8.1 *$ & $<0.001$ \\
\hline VMO & $-38.5 \pm 3.7^{*}$ & $<0.001$ & $-38.8 \pm 3.6^{*}$ & $<0.001$ & $-36.5 \pm 3.9^{*}$ & $<0.001$ \\
\hline
\end{tabular}

1

2 